\title{
The Post-Implantation Electrocardiogram Predicts Clinical Response to Cardiac Resynchronization Therapy
}

\author{
Edward Coverstone, MD1 , Justin Sheehy, MD $^{2}$, Robert E Kleiger, MD $^{1}$, and Timothy W \\ Smith, DPhil, MD ${ }^{1}$ \\ ${ }^{1}$ Washington University School of Medicine, Department of Medicine, Cardiovascular Division, \\ Saint Louis, Missouri \\ ${ }^{2}$ Washington University School of Medicine, Department of Medicine, Saint Louis, Missouri
}

\section{Abstract}

Background-Biventricular pacing for cardiac resynchronization therapy (CRT) is intended to improve left ventricular function by coordinating systolic activity of the septum and free walls. Optimal resynchronization should be manifested by 12-lead electrocardiogram (ECG) patterns consistent with resynchronized activation, a tall ( $\geq 4 \mathrm{~mm}$ ) $\mathrm{R}$ wave in $\mathrm{V} 1$ and predominant negative deflection in lead I (RV1SI). We investigated whether the presence or absence of RV1SI predicts heart failure outcomes within one year of CRT implant.

Methods-Two independent physicians reviewed the paced ECG of 213 patient post-CRT device implantation with disputes resolved by a third reviewer. The primary endpoints of all-cause death, unplanned hospitalization, left-ventricular assist device implant, or transplant within a one year follow-up were blindly adjudicated according to standard definitions. Groups were compared via Kaplan-Meier estimates and Cox proportional hazards models to determine association with event-free survival.

Results-Among CRT patients post-implantation, 56 (26.3\%) exhibited the RV1SI pattern on ECG. Patients with the RV1SI pattern were significantly less likely to achieve the primary endpoint as compared to patients without the RV1SI pattern (33.9 v. 52.2\%; Log Rank p=0.022). This difference was driven by a significantly lower risk for unplanned hospitalization among patients with the RV1SI pattern ( $\mathrm{HR}=0.510$; CI $[0.298,0.876])$. The predictive value remained after adjustment for potential confounders $(\mathrm{p}=0.004)$.

Conclusions-The 12-lead ECG post-implantation predicts clinical outcomes of biventricular pacing. Such prediction may be useful in predicting the need for alternative or advanced heart failure therapies. Further study into ECG patterns may help to prospectively guide CRT.

Correspondence to: Edward Coverstone, Washington University School of Medicine, 660 S. Euclid Ave., Box 8086, Saint Louis, Missouri 63110, FAX: 314-362-4619, ecoverst@dom.wustl.edu.

The content is solely the responsibility of the authors and does not necessarily represent the official view of the NIH.

Disclosure: Dr. Coverstone receives training grant support by National Institutes of Health, National Research Service Award 5-T32HL07081-38, from the National Heart, Lung, and Blood Institute. The coauthors report no additional financial disclosure or conflict of interest. 


\section{Keywords}

Biventricular Pacing; Cardiac Resynchronization Therapy; Electrocardiogram; Congestive Heart Failure

\section{INTRODUCTION}

Cardiac resynchronization therapy (CRT) utilizes an implantable biventricular (BiV) pacemaker to pace the left and right ventricle in order to coordinate systolic motion of the left ventricular septum and free wall. In heart failure patients with left bundle branch block (LBBB), CRT has proven benefit over dual chamber implantable cardiac devices or medical therapy alone. $(1 ; 2)$ The 2012 ACCF/AHA/HRF guidelines for device-based therapy of cardiac rhythm abnormalities include a Class I indication for CRT in heart failure patients with an underlying LBBB, New York Heart Association Class II-IV symptoms, and QRS duration greater than $150 \mathrm{~ms} .(3)$

Approximately $30-40 \%$ of heart failure patients do not show clinical improvement to CRT (non-responders),(4) suggesting the importance of differentiating responders from nonresponders prior to implantation. While many pre-implantation variables based upon clinical classifications, electrocardiogram (ECG) parameters, and imaging have been identified that correlate with clinical outcomes;(5-7) few studies have found post-implantation parameters to predict clinical response.(8-11) However, the ability to predict response to CRT even after implantation is a valuable tool that may allow optimization of programming,(12) tailoring of patient-centered heart failure therapies, or prevention of harm from inappropriate device therapy.(13)

Electrocardiographically, CRT most commonly results in a QRS that represents fusion of the wave fronts initiated from the right ventricular and left ventricular leads. Successful biventricular or left ventricular pacing from a lateral or posterior lead position should result in a QRS that is directed anteriorly, rightward, and superiorly.(14) This typically will produce prominent $\mathrm{R}$ waves in V1, V2, and aVR; qS waves in I and aVL; and a northwest axis in an appropriately paced bi-ventricular system.(15-17) This pattern may be simplified to a predominant negative deflection ( $\mathrm{S}$ wave or $\mathrm{qS}$ ) in lead I and $\mathrm{R}$ wave greater than or equal to $4 \mathrm{~mm}$ in V1 which we describe as an RV1SI pattern (Figure 1). When resynchronization fails, it may be due to: suboptimal placement of the left ventricular lead, suboptimal timing of LV and/or RV pacing, or marked conduction abnormality from the pacing sites, which may be reflected electrocardiographically.(18)

We hypothesized that the presence or absence of the RV1SI pattern on the post-implantation ECG could predict clinical congestive heart failure outcomes at one year. We retrospectively examined post-implantation ECGs from over a six year time period from a multicenter database. As demonstrated below, these ECG parameters after implantation of the device significantly correlate with and predict the clinical course of heart failure at one year. 


\section{METHODS}

\section{Population and Study Design}

The study was approved by the Institutional Review Board of the Human Research Protection Office at Washington University in Saint Louis. Patients were identified by procedural ICD-9 codes via CIDER (Clinical Investigation Data Exploration Repository) searchable database. We identified 2,162 patients from January 1, 2006 to January 1, 2012, who underwent $\mathrm{BiV}$ pacemaker implantation in the Barnes-Jewish Health Care Network. Implantations occurred at five separate medical centers (Barnes-Jewish, Christian Northeast, Missouri Baptist, Boone County, and Alton Regional) representing a diverse cohort of community and academic hospitals in the Midwest United States. A random cohort of 250 patients was selected utilizing a random number generator of which 213 patients fit the inclusion and exclusion criteria for data analysis.

Enrollment criteria required (1) age greater than 18 years old and (2) successful implantation of a cardiac resynchronization device or generator change. Patients were excluded if there was a lack of electrocardiogram during the follow-up period. Electrocardiograms for analysis were prioritized as (1) closest prior to discharge post-implantation, (2) outpatient clinic post-implantation, or (3) inpatient hospitalization within the follow-up period. ECG interpretation was performed by two independent physician reviewers with disputes resolved by a third reviewer. Baseline patient characteristics and outcomes were collected by retrospective chart review. Primary outcomes were adjudicated by an initial chart review then verified by an independent, blinded physician reviewer according to stated definitions. All data was collected using REDCAP survey tool.(19)

\section{Outcomes and Definitions}

The primary outcome was a combination of all-cause mortality, unplanned hospitalization, and advanced heart failure therapy at a follow-up of one year. Unplanned hospitalization was defined as a non-scheduled admission greater than 24 hours for a cardiovascular or noncardiovascular diagnosis or complication from cardiac procedure. Planned hospitalizations for further electrophysiology procedures such as lead revisions, timing alterations, or other pacemaker modifications were excluded to avoid confounding. Advanced heart failure therapy was defined as surgical implantation of left ventricular assist device or cardiac transplantation. Secondary outcomes included the individual components of the combined outcome as well as change in left-ventricular ejection fraction. Change in ejection fraction was recorded from available reported cardiac magnetic resonance imaging, echocardiography, nuclear study, or cardiac catheterization data within a one year period from CRT device implantation. Ejection fraction post device implantation was collected no earlier than one month post procedure.

Patient demographics, patient history, electrocardiographic patterns, and pacemaker history were defined as in the ACC/AHA/HRS Clinical Data Standards for Electrocardiographic Studies and Procedures.(20)

Reviewers collected the following electrocardiographic data from the post-implantation ECG: QRS duration, QRS axis, S wave (described broadly as the presence of primarily 
negative deflection in lead I), and height of R-wave in lead V1. Electrocardiograms that did not reveal clear ventricular pacing were excluded. Underlying bundle branch block pattern was determined from pre-implantation ECG by the implanting physician and recorded in the National Cardiovascular Data Registry-Implantable Cardioverter Defibrillator Registry or when missing by ECG reviewer according to AHA/ACCF/HRS definitions.(21)

\section{Statistical Analysis}

The sample size was estimated based on results of a pilot study of 50 consecutive patients undergoing BIV insertion. The primary outcome occurred in $17.5 \%$ of patients with RV1SI findings as compared with $51.5 \%$ of the patients without the ECG findings. Estimating the true difference in proportion of these groups to be smaller; we powered our study to an incidence of $30 \%$ in the ECG findings group and $45 \%$ in the findings absent group. Given a power of $90 \%$ and alpha of 0.05 , we estimated to find such a difference in equality of two proportions would require a sample size of approximately 200 patients.(22) Patients from the pilot study were excluded from the final study cohort.

Statistical analysis was performed using IBM SPSS Statistics 22. Baseline and clinical characteristics were compared as follows. Differences in categorical data were reported as frequencies then compared using chi-square or Fisher's exact tests, as appropriate.

Continuous variables are reported as means \pm standard deviation with differences between groups tested using independent samples t-tests. Due to skewed distribution, age and ejection fraction are reported as median \pm interquartile range and differences analyzed with non-parametric testing. Primary outcomes were reported as an initial incidence within the defined time period of one year and analyzed via chi-square tests. Kaplan-Meier curves were plotted for the primary composite outcome and Cox proportional hazards models created for unadjusted and adjusted analysis. All statistical tests were 2-sided using a p-value of less than 0.05 to indicate statistical significance.

\section{RESULTS}

\section{Study population and baseline characteristics}

213 patients were enrolled who fit the inclusion and exclusion criteria. The study cohort underwent BiV pacemaker implantations from February 2006 to December 2012. The baseline characteristics for the study cohort are shown in Table 1 . The population was primarily male $(60.6 \%)$ with a median age of 69 years \pm 18 . LBBB $(57.7 \%)$ was largely the predominant underlying IVCD although a considerable number of patients were characterized as right bundle branch block (RBBB) (12.7\%) or indeterminate $(29.6 \%)$. The median left ventricular ejection fraction (LVEF) was $25.7 \% \pm 14.2$.

There were 56 patients with the RV1SI ECG pattern as compared with 157 patients without the optimal ECG pattern. The proportion of patients with the individual RV1 (37.6\%) and SI (55.9\%) components was similar to what has been previously described in the literature.(11) Patients with the optimal RV1SI pattern were similar in baseline characteristics to patients without (Table 2). There was no significant difference in age, gender, prior medical history, underlying QRS pattern, or ejection fraction. However, there was a statistically significant 
lower proportion of patients with non-ischemic cardiomyopathy patients who exhibited the clinical electrocardiographic findings ( $26.8 \%$ v. $45.9 \%, p=0.013)$.

\section{Outcomes}

The presence of the RV1SI pattern on post-implantation ECG was significantly associated with freedom from the primary combined outcome of death, unplanned hospitalization, or advanced heart failure therapy at one year $(33.9 \%$ v $52.2 \%, \mathrm{p}=0.019)$. The primary outcomes were plotted on Kaplan-Meier curves (Figure 2) showing the ECG pattern significantly predicted freedom from the primary outcomes to one year ( $\log \operatorname{Rank} \mathrm{p}=0.022)$. Of the primary outcomes, only unplanned hospitalization was independently associated with the electrocardiographic findings and carried much of the weight of the hazard reduction (HR 0.510, 95\%CI [0.298,0.876]; $\mathrm{p}=0.015$ ). Cardiovascular hospitalizations accounted for 72.8\% unplanned hospitalizations and were similarly less frequent in the RV1SI group $(23.2 \%$ v $34.4 \%)$. There was no statistical difference in the secondary outcome of change in left ventricular ejection fraction $(7.82 \%$ v. $5.51 \%, \mathrm{p}=0.365)$.

As a diagnostic test, the presence of the RV1SI pattern was specific for predicting clinical CRT benefit as defined by freedom from events at one year (specificity $81.2 \%$, sensitivity $33.0 \%)$. Patients with the RV1SI pattern were 1.7 times more likely (LR+ 1.76, [1.08,2.85]) to avoid adverse outcomes at one year.

\section{Adjustments}

In prior CRT studies, female sex, non-ischemic origin, left-bundle branch block, and QRS duration have been shown to be associated with favorable echocardiographic response (>10\% reduction in LVEDv) and clinical outcomes of heart failure and death (23) and therefore were identified for prespecified adjusted analysis. Paced QRS duration was also found to be significantly longer among patients with the RV1SI pattern (157 v. 143, $\mathrm{p}=0.001$ ) and was therefore considered for inclusion into the adjusted model. However, their addition did not significantly change the ability of the RV1SI pattern to predict the primary outcome (HR 0.465, p=0.004; Table 4). Furthermore, other potential ECG variables such as paced QRS duration, QRS axis, or R-wave height alone did not predict the primary outcome.

A growing amount of evidence recognizes a negative influence of atrial fibrillation on clinical CRT response.(24-26) Atrial fibrillation without rate control or AV nodal ablation may result in native conduction through the AV node and His Purkinje system which may be reflected as pseudo-fusion on ECG.(27) Such a pattern would unlikely reflect an optimal response to CRT. To exclude the possibility that the effectiveness of RV1SI pattern was related to a signal from atrial fibrillation and pseudofusion alone, we stratified its predictive ability among this subgroup. Among patients without a diagnosis of atrial fibrillation or flutter, the RV1SI pattern remained predictive with the primary outcome occurring in $19.0 \%$ $(n=4)$ of patients with the ECG pattern as compared with $43.1 \%(n=28)$ of patients without $(\mathrm{p}=0.048)$. 


\section{Non-left bundle branch block}

In our cohort, there were a significant number of patients $(n=27)$ with underlying RBBB pattern who underwent CRT implantation, representative of the limitations of data regarding non-LBBB and implantation during the study time period of 2006-2012. Such a cohort may be representative of current CRT patients in the clinical setting. As an exploratory analysis, these patients were analyzed to determine if the RV1SI pattern predicted outcomes among this subgroup. Among all non-LBBB block patients $(n=90)$, the presence of the RV1SI pattern was strongly predictive of decreased risk for the primary outcome at one year (HR 0.304, $\mathrm{p}=0.006$ ). In particular, RBBB patients with the optimal CRT pacing pattern were significantly less likely to undergo the primary composite outcome $(11.1 \% \mathrm{v} 72.2 \%$, $\mathrm{p}=0.003)$. The RV1SI pattern was an extremely specific ( $92.9 \%$ specificity, $61.5 \%$ sensitivity) predictor of freedom from the primary outcome in the RBBB subgroup.

\section{DISCUSSION}

We present the description of a simple electrocardiographic algorithm utilizing the paced QRS complex that is associated with clinical outcomes post-cardiac resynchronization therapy. To our knowledge, no prior study has successfully utilized the biventricular paced QRS morphology to predict clinical outcomes in CRT.

Barold et al. first described the use of a similar algorithm to define the electrical activation from biventricular pacing. They described the typical ECG of biventricular pacing with a dominant $\mathrm{R}$ wave in $\mathrm{V} 1$ and the presence of right axis deviation, establishing their definitions for a fused QRS complex as associated with optimal CRT pacing.(16) Ammann et al. similarly published an algorithm based upon an R:S ratio in leads I and V1 to predict loss of LV lead capture with high sensitivity and specificity. The 54 patient cohort was limited to patients with left-bundle branch block and did not attempt correlation with heart failure outcomes. Their study effectively showed the utility of the paced QRS complex to predict lead failure.(12) Kobe et al. evaluated the peri-procedural ECG during CRT implantation and developed an algorithm of left ventricle paced QRS width minus right ventricle paced $\mathrm{QRS}$ width to be predictive $(\mathrm{p}=0.02)$ of clinical response as defined by improvement in NYHA class at 3 month follow-up.(28)

Sweeney et al. compared the pre and post implantation ECG of 202 patients with underlying LBBB and identified variables associated with a $\geq 10 \%$ reduction in LV systolic volumes. Their multivariate regression model identified increasing $\mathrm{R}$ wave height in V1 and V2 as well as a shift from left axis deviation to right axis deviation as associated with echocardiographic response to CRT.(11) These findings coincide with the clinical association of the RV1SI pattern with hard clinical endpoints identified in our study; reinforcing the appropriateness of the RV1SI pattern and providing mechanistic insight.

Prior studies have also examined the utility of the QRS duration to predict clinical outcomes. Kiani et al evaluated 112 patients post-CRT implantation and utilized a QRS shortening of $1 \mathrm{~ms}$ to define reverse electrical remodeling. They found no association between QRS duration and survival in their study.(29) Tereshchenko et al. found in a cohort of 69 patients that a shortening of the underlying non-paced QRS was associated with a fourfold decrease 
in the risk of death or sustained ventricular tachycardia.(30) Shortening of the paced QRS, however, may not accurately represent electrical or mechanical resynchronization. In our population, the duration of the paced QRS did not correlate with the primary outcomes of death, unplanned hospitalization, or advanced heart failure therapy; nor did it correlate with the secondary outcome of improvement in LVEF.

Prior investigations have focused on the pre-implantation ECG to predict outcomes. Posthoc analyses from multiple randomized trials have found baseline electrocardiographic QRS morphology predicts clinical response to cardiac resynchronization therapy. Results from MADIT-CRT in over 1800 heart failure patients with LVEF $</=30 \%$ found LBBB pattern was associated with an odds ratio of $0.47(\mathrm{p}<0.001)$ for the clinical composite outcome of sustained ventricular fibrillation, ventricular tachycardia, or death. Interestingly, in the subgroup of patients with non-LBBB IVCD, there was a non-statistically significant trend towards harm.(31) Recently, Goldberg et al. confirmed the importance of underlying LBBB in their long-term follow-up into 854 of the original MADIT-CRT cohort. Not only did they find a survival advantage for CRT-defibrillator over ICD alone in patients with underlying LBBB; but furthermore, the presence of CRT in patients without LBBB was significantly associated with increased mortality (HR 1.57, 95\% CI 1.03-2.39, p=0.04).(32) Given this data, there may be concern given the lack of benefit and potential for harm for CRT in nonLBBB patients. Previous clinical practice, however, has resulted in patients with RBBB pathology receiving devices making longitudinal follow-up decisions concerning lead revisions, generator changes, or cessation of therapy rather challenging. In the RBBB subgroup, our data shows that the RV1SI pattern appears to be extremely predictive of clinical outcomes with high sensitivity. Therefore, the use of this algorithm may help risk stratify non-LBBB patients in whom discontinuation of CRT therapy is being considered.

Our study has several limitations. First, our study was performed by retrospective observational cohort study and therefore may have limitation in reliability for long-term follow-up as can be performed in a prospective observation or randomized trial setting. However, the Barnes-Jewish electronic health record presented a readily searchable database with reliable patient follow-up. Second, the electrocardiographic algorithm may not be the most predictive algorithm available of the paced QRS complex. The design of our algorithm was by a combination of prior CRT electrocardiographic descriptions as well as subjective ECG reader experience, then validated in a small 50 patient sample size. Other electrocardiographic parameters may be more sensitive or specific for optimal biventricular pacing; however, the definitions of such parameters are beyond the scope of this study. Furthermore, clinical tools available to the electrophysiologist such as radiographic lead position or device interrogation (percent CRT pacing and atrial fibrillation burden) were not addressed as potential confounders. Therefore, the RV1SI pattern does not take precedence over these known predictors of clinical outcomes in CRT but rather may prompt such further investigations. Finally, the observational nature of the study prevents the conclusion of treatment effect. While one cannot definitively infer that changing procedural characteristics or timing characteristics based on the algorithm will improve clinical outcomes, we find this hypothesis generating for prospective investigation. 
The causes for an abnormal biventricular pacing pattern are diverse and include both modifiable and non-modifiable clinical circumstances. They include suboptimal left ventricular lead placement or failure, latency of conduction due to disease of the ventricle, and fusion with conducted QRS complex.(16) Some defects may be corrected by electrophysiological or clinical interventions in order to achieve optimal biventricular pacing. Simple modifications such as shortening AV delay or altering V-V programming have been shown to optimize the paced QRS pattern which, in turn, correlates with echocardiographic optimization.(33) Pseudofusion with conducted complexes (and ineffective pacing) may be relieved by medically increasing AV nodal blockade or, if necessary, nodal ablation. Therefore, utilization of an ECG pattern that correlates with clinical outcomes not only has utility in risk prediction but may have potential in directing future investigation into clinical treatments and interventions.

\section{CONCLUSIONS}

In conclusion, the paced ECG post-CRT implantation is an important tool that predicts clinical outcomes in heart failure patients. The presence of an RV1SI pattern is strongly associated with freedom from the primary combined outcomes of death, unplanned hospitalization, or advanced heart failure therapy at one year. This simple tool may be used to not only predict clinical outcomes and risk stratify patients, but provide an algorithm for further investigation into the utility of ECG-guided CRT therapy.

\section{Acknowledgments}

Funding: Research reported in this publication was supported by the Washington University Institute of Clinical and Translational Sciences grant UL1TR000448 from the National Center for Advancing Translational Sciences (NCATS) of the National Institutes of Health (NIH).

Funding for the Clinical Investigation Data Exploration Repository (CIDER) and REDCAP survey tool at Washington University in Saint Louis is granted through NIH CTSA Grant \# UL1 TR000448.

\section{ABBREVIATIONS}

$\begin{array}{ll}\text { BiV } & \text { Biventricular } \\ \text { CRT-D } & \text { Implantable Cardioverter-Defibrillator Device } \\ \text { CRT } & \text { Cardiac Resynchronization Therapy } \\ \text { ECG } & \text { Electrocardiogram } \\ \text { LBBB } & \text { Left Bundle Branch Block } \\ \text { LVEDv } & \text { Left Ventricular End-Diastolic Volume } \\ \text { LVEF } & \text { Left Ventricular Ejection Fraction } \\ \text { RBBB } & \text { Right Bundle Branch Block }\end{array}$




\section{References}

1. Bardy GH, Lee KL, Mark DB, Poole JE, Packer DL, Boineau R, Domanski M, et al. Amiodarone or an implantable cardioverter-defibrillator for congestive heart failure. The New England journal of medicine. 2005; 352:225-237. [PubMed: 15659722]

2. Moss AJ, Hall WJ, Cannom DS, Klein H, Brown MW, Daubert JP, Estes NA 3rd, et al. Cardiacresynchronization therapy for the prevention of heart-failure events. The New England journal of medicine. 2009; 361:1329-1338. [PubMed: 19723701]

3. Tracy CM, Epstein AE, Darbar D, DiMarco JP, Dunbar SB, Estes NA 3rd, Ferguson TB Jr, et al. 2012 ACCF/AHA/HRS focused update of the 2008 guidelines for device-based therapy of cardiac rhythm abnormalities: a report of the American College of Cardiology Foundation/American Heart Association Task Force on Practice Guidelines and the Heart Rhythm Society. [corrected]. Circulation. 2012; 126:1784-1800. [PubMed: 22965336]

4. Chung ES, Leon AR, Tavazzi L, Sun JP, Nihoyannopoulos P, Merlino J, Abraham WT, et al. Results of the Predictors of Response to CRT (PROSPECT) trial. Circulation. 2008; 117:26082616. [PubMed: 18458170]

5. Boidol J, Sredniawa B, Kowalski O, Szulik M, Mazurek M, Sokal A, Pruszkowska-Skrzep P, et al. Many response criteria are poor predictors of outcomes after cardiac resynchronization therapy: validation using data from the randomized trial. Europace: European pacing, arrhythmias, and cardiac electrophysiology: journal of the working groups on cardiac pacing, arrhythmias, and cardiac cellular electrophysiology of the European Society of Cardiology. 2013; 15:835-844.

6. Ellims AH, Pfluger H, Elsik M, Butler MJ, Hare JL, Taylor AJ. Utility of cardiac magnetic resonance imaging, echocardiography and electrocardiography for the prediction of clinical response and long-term survival following cardiac resynchronisation therapy. The international journal of cardiovascular imaging. 2013; 29:1303-1311. [PubMed: 23568562]

7. Brenyo A, Zareba W. Prognostic significance of QRS duration and morphology. Cardiology journal. 2011; 18:8-17. [PubMed: 21305480]

8. Sonne C, Bott-Flugel L, Hauck S, Hadamitzky M, Lesevic H, Demetz G, Braun D, et al. Threedimensional echocardiographic optimization improves outcome in cardiac resynchronization therapy compared to ECG optimization: a randomized comparison. Pacing and clinical electrophysiology: PACE. 2014; 37:312-320. [PubMed: 24164640]

9. Auricchio A, Prinzen FW. Non-responders to cardiac resynchronization therapy: the magnitude of the problem and the issues. Circulation journal: official journal of the Japanese Circulation Society. 2011; 75:521-527. [PubMed: 21325727]

10. Lecoq G, Leclercq C, Leray E, Crocq C, Alonso C, de Place C, Mabo P, et al. Clinical and electrocardiographic predictors of a positive response to cardiac resynchronization therapy in advanced heart failure. European heart journal. 2005; 26:1094-1100. [PubMed: 15728648]

11. Sweeney MO, van Bommel RJ, Schalij MJ, Borleffs CJW, Hellkamp AS, Bax JJ. Analysis of Ventricular Activation Using Surface Electrocardiography to Predict Left Ventricular Reverse Volumetric Remodeling During Cardiac Resynchronization Therapy. Circulation. 2010; 121:626634. [PubMed: 20100970]

12. Ammann P, Sticherling C, Kalusche D, Eckstein J, Bernheim A, Schaer B, Osswald S. An electrocardiogram-based algorithm to detect loss of left ventricular capture during cardiac resynchronization therapy. Annals of internal medicine. 2005; 142:968-973. [PubMed: 15968011]

13. Singh JP, Klein HU, Huang DT, Reek S, Kuniss M, Quesada A, Barsheshet A, et al. Left ventricular lead position and clinical outcome in the multicenter automatic defibrillator implantation trial-cardiac resynchronization therapy (MADIT-CRT) trial. Circulation. 2011; 123:1159-1166. [PubMed: 21382893]

14. Ploux S, Bordachar P, Deplagne A, Mokrani B, Reuter S, Laborderie J, Garrigue S, et al. Electrocardiogram-based algorithm to predict the left ventricular lead position in recipients of cardiac resynchronization systems. Pacing and clinical electrophysiology: PACE. 2009; 32(Suppl 1):S2-7. [PubMed: 19250094]

15. Barold SS, Herweg B. Usefulness of the 12-lead electrocardiogram in the follow-up of patients with cardiac resynchronization devices. Part II. Cardiology journal. 2011; 18:610-624. [PubMed: 22113748] 
16. Barold SS, Herweg B. Usefulness of the 12-lead electrocardiogram in the follow-up of patients with cardiac resynchronization devices. Part I. Cardiology journal. 2011; 18:476-486. [PubMed: 21947982]

17. Giudici MC, Tigrett DW, Carlson JI, Lorenz TD, Paul DL, Barold SS. Electrocardiographic patterns during: pacing the great cardiac and middle cardiac veins. Pacing and clinical electrophysiology: PACE. 2007; 30:1376-1380. [PubMed: 17976102]

18. !!! INVALID CITATION !!!

19. Harris PA, Taylor R, Thielke R, Payne J, Gonzalez N, Conde JG. Research electronic data capture (REDCap)--a metadata-driven methodology and workflow process for providing translational research informatics support. Journal of biomedical informatics. 2009; 42:377-381. [PubMed: 18929686]

20. Buxton AE, Calkins H, Callans DJ, DiMarco JP, Fisher JD, Greene HL, et al. American College of Cardiology/American Heart Association Task Force on Clinical Data S. ACC/AHA/HRS 2006 key data elements and definitions for electrophysiological studies and procedures: a report of the American College of Cardiology/American Heart Association Task Force on Clinical Data Standards (ACC/AHA/HRS Writing Committee to Develop Data Standards on Electrophysiology). Circulation. 2006; 114:2534-2570. [PubMed: 17130345]

21. Surawicz B, Childers R, Deal BJ, Gettes LS, Bailey JJ, Gorgels A, Hancock EW, et al. AHA/ ACCF/HRS recommendations for the standardization and interpretation of the electrocardiogram: part III: intraventricular conduction disturbances: a scientific statement from the American Heart Association Electrocardiography and Arrhythmias Committee, Council on Clinical Cardiology; the American College of Cardiology Foundation; and the Heart Rhythm Society. Endorsed by the International Society for Computerized Electrocardiology. J Am Coll Cardiol. 2009; 53:976-981. [PubMed: 19281930]

22. Lenth, R. Java Applets for Power and Sample Size. 2006-09. Computer Software

23. Goldenberg I, Moss AJ, Hall WJ, Foster E, Goldberger JJ, Santucci P, Shinn T, et al. Predictors of response to cardiac resynchronization therapy in the Multicenter Automatic Defibrillator Implantation Trial with Cardiac Resynchronization Therapy (MADIT-CRT). Circulation. 2011; 124:1527-1536. [PubMed: 21900084]

24. Gasparini M, Leclercq C, Lunati M, Landolina M, Auricchio A, Santini M, Boriani G, et al. Cardiac Resynchronization Therapy in Patients With Atrial Fibrillation The CERTIFY Study (Cardiac Resynchronization Therapy in Atrial Fibrillation Patients Multinational Registry). JACC: Heart Failure. 2013; 1:500-507. [PubMed: 24622002]

25. Gasparini M, Regoli F, Galimberti P, Ceriotti C, Cappelleri A. Cardiac resynchronization therapy in heart failure patients with atrial fibrillation. Europace: European pacing, arrhythmias, and cardiac electrophysiology: journal of the working groups on cardiac pacing, arrhythmias, and cardiac cellular electrophysiology of the European Society of Cardiology. 2009; 11:v82-v86.

26. Molhoek SG, Bax JJ, Bleeker GB, Boersma E, van Erven L, Steendijk P, van der Wall EE, et al. Comparison of response to cardiac resynchronization therapy in patients with sinus rhythm versus chronic atrial fibrillation. The American Journal of Cardiology. 2004; 94:1506-1509. [PubMed: 15589005]

27. ECG Response: August 26, 2014. Circulation. 2014; 130:799-800. [PubMed: 25156916]

28. Kobe J, Dechering DG, Rath B, Reinke F, Monnig G, Wasmer K, Eckardt L. Prospective evaluation of electrocardiographic parameters in cardiac resynchronization therapy: detecting nonresponders by left ventricular pacing. Heart rhythm: the official journal of the Heart Rhythm Society. 2012; 9:499-504. [PubMed: 22079557]

29. Kiani J, Agarwal SK, Kamireddy S, Adelstein E, Saba S. Relationship of electromechanical remodeling to survival rates after cardiac resynchronization therapy. Texas Heart Institute journal/ from the Texas Heart Institute of St. Luke's Episcopal Hospital, Texas Children's Hospital. 2013; 40:268-273.

30. Tereshchenko LG, Henrikson CA, Stempniewicz P, Han L, Berger RD. Antiarrhythmic effect of reverse electrical remodeling associated with cardiac resynchronization therapy. Pacing and clinical electrophysiology: PACE. 2011; 34:357-364. [PubMed: 21091740]

31. Zareba W, Klein H, Cygankiewicz I, Hall WJ, McNitt S, Brown M, Cannom D, et al. Effectiveness of Cardiac Resynchronization Therapy by QRS Morphology in the Multicenter Automatic 
Defibrillator Implantation Trial-Cardiac Resynchronization Therapy (MADIT-CRT). Circulation. 2011; 123:1061-1072. [PubMed: 21357819]

32. Goldenberg I, Kutyifa V, Klein HU, Cannom DS, Brown MW, Dan A, Daubert JP, et al. Survival with cardiac-resynchronization therapy in mild heart failure. The New England journal of medicine. 2014; 370:1694-1701. [PubMed: 24678999]

33. Herweg B, Ali R, Ilercil A, Madramootoo C, Cutro R, Weston MW, Barold SS. Site-specific differences in latency intervals during biventricular pacing: impact on paced QRS morphology and echo-optimized V-V interval. Pacing and clinical electrophysiology: PACE. 2010; 33:1382-1391. [PubMed: 20735713] 


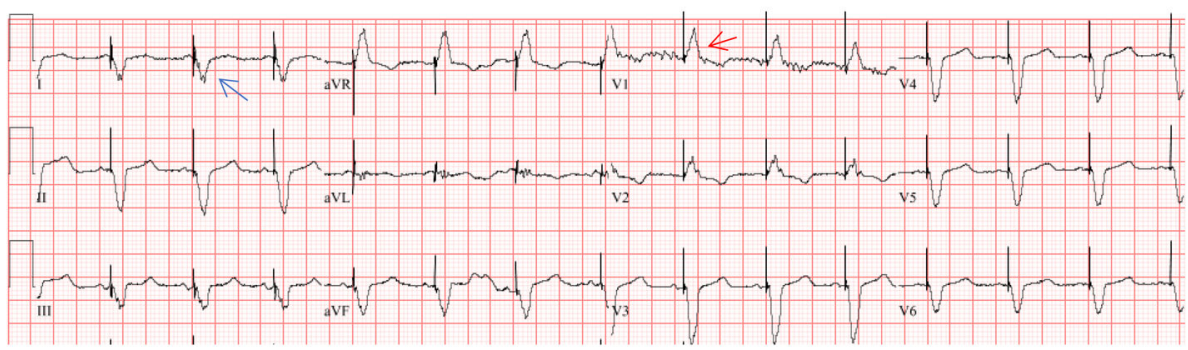

A)

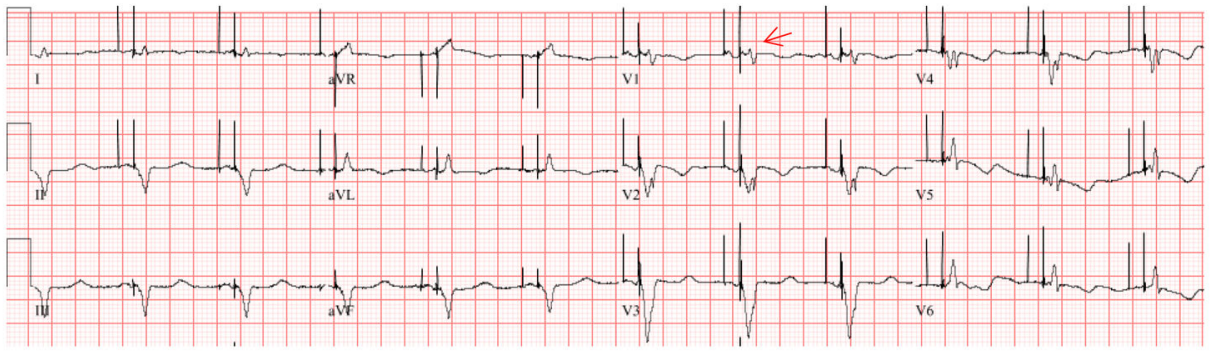

B)

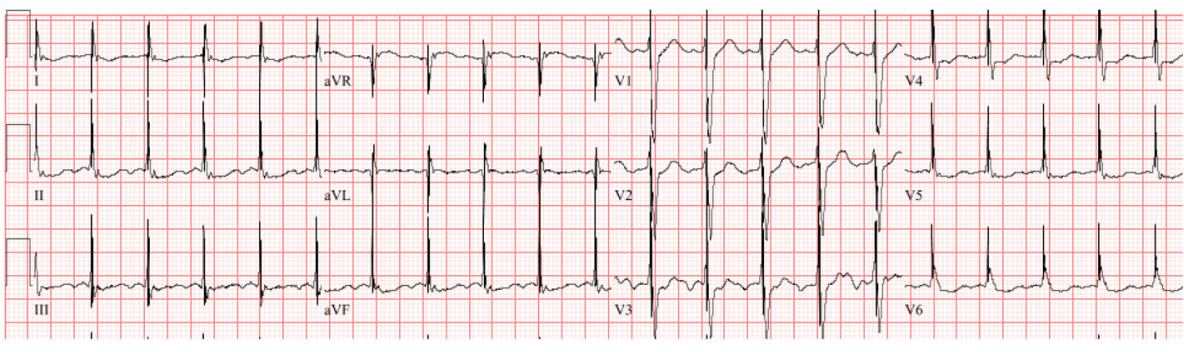

C)

Figure 1.

A) The ECG of a 62 year-old male with a past medical history of coronary artery disease and ischemic cardiomyopathy post-implantation of a Medtronic Concerto Biventricular, Implantable Cardioverter-Defibrillator Device (CRT-D) exhibits the optimal RV1SI pattern of tall ( $₫ 4 \mathrm{~mm}) \mathrm{R}$ wave in V1 (Red Arrow), and predominant negative deflection, represented by a qS in lead I (Blue Arrow). The patient went on to have improvement in his NYHA class and freedom from unplanned hospitalization one year post-implantation. B) The ECG of a 65 year-old male with a similar history of ischemic heart disease postimplantation of Medtronic Concerto Model CRT-D with poor R wave height in V1 (Red Arrow). He was hospitalized for heart failure exacerbation 4 months post-implantation and died 5 months post-implantation. C) The ECG of a 60 year-old male with ischemic cardiomyopathy post Medtronic Concerto II CRT-D with pseudofusion of native conduction resulting in a suboptimal ECG pattern. 


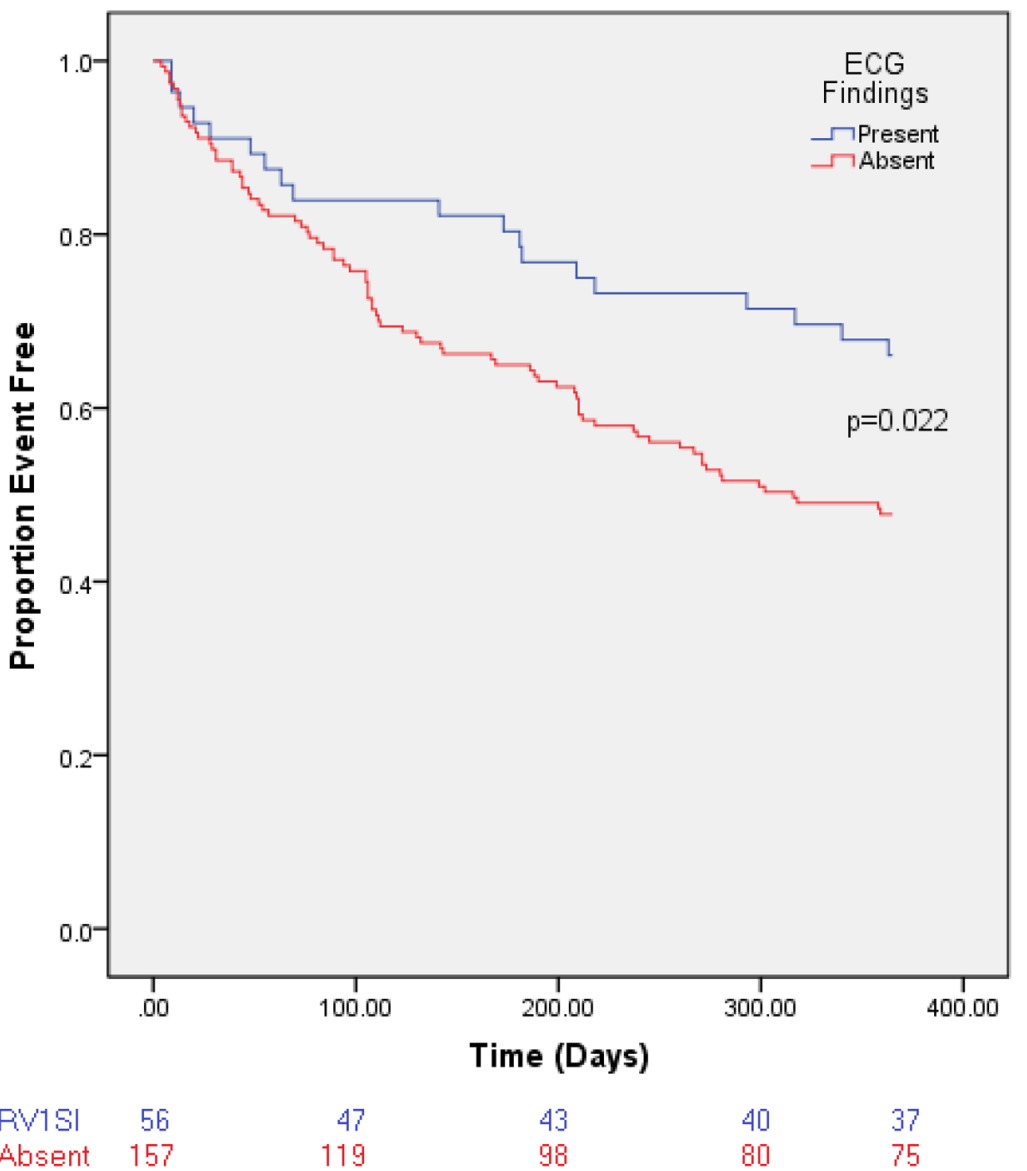

Figure 2.

Kaplan-Meier Curve for the primary composite endpoint at one year with separation of the curves at 365 days. Kaplan-Meier test (log rank) test of equality showed significant difference between groups ( $\mathrm{p}=0.022$ ). Adjusted analysis by Cox Regression including age, gender, etiology for cardiomyopathy, underlying bundle branch block, and ejection fraction as variables, attested a difference between groups $(\mathrm{p}=0.020)$. 


\section{Table 1}

Baseline characteristics including age, gender, medical history, etiology of cardiomyopathy, underlying bundle branch block, and ejection fraction.

\begin{tabular}{|lrr|}
\hline Baseline Characteristics & N=213 & \% or IQ Range \\
\hline Age, years & 69.0 & 18.0 \\
\hline Gender & & \\
\hline Male & 129 & $60.6 \%$ \\
\hline Female & 84 & $39.4 \%$ \\
\hline Hypertension & 173 & $81.2 \%$ \\
\hline Hyperlipidemia & 168 & $78.9 \%$ \\
\hline Diabetes Mellitus & 102 & $47.9 \%$ \\
\hline Smoking History & 88 & $41.3 \%$ \\
\hline Peripheral Arterial Disease & 41 & $19.2 \%$ \\
\hline Atrial Fibrillation/Flutter & 127 & $59.6 \%$ \\
\hline Chronic Kidney Disease & & \\
\hline (Stage III-IV) & 95 & $44.6 \%$ \\
\hline Cardiomyopathy & & \\
\hline Ischemic & 112 & $52.6 \%$ \\
\hline Non-Ischemic & 88 & $41.3 \%$ \\
\hline Mixed & 13 & $6.1 \%$ \\
\hline QRS Morphology & & \\
\hline Left Bundle Branch Block & 123 & $57.7 \%$ \\
\hline Right Bundle Branch Block & 27 & $12.7 \%$ \\
\hline Indeterminate & $60.2 \%$ \\
\hline Ejection Fraction, \% & \\
\hline
\end{tabular}


Table 2

Differences in clinical characteristics among patients with RV1SI ECG findings compared to those without the ECG findings.

\begin{tabular}{|c|c|c|c|}
\hline Clinical Characteristics & ECG Findings Present $(n=56)$ & ECG Findings Absent (n=157) & P Value \\
\hline Age, years ${ }^{*}$ & $70.5(19)$ & $67.5(18)$ & $\mathrm{p}=0.192$ \\
\hline Gender & & & $\mathrm{p}=0.353$ \\
\hline Male & $31(55.4 \%)$ & $98(62.4 \%)$ & \\
\hline Female & $25(44.6 \%)$ & $59(37.6 \%)$ & \\
\hline Hypertension & $49(87.5 \%)$ & $124(79.0 \%)$ & $\mathrm{p}=0.161$ \\
\hline Hyperlipidemia & $45(80.4 \%)$ & $123(78.3 \%)$ & $\mathrm{p}=0.353$ \\
\hline Diabetes Mellitus & $28(50.0 \%)$ & $74(47.1 \%)$ & $\mathrm{p}=0.712$ \\
\hline Smoking History & $23(41.1 \%)$ & $65(41.4 \%)$ & $\mathrm{p}=0.966$ \\
\hline Peripheral Arterial Disease & $11(19.6 \%)$ & $30(19.1 \%)$ & $\mathrm{p}=0.931$ \\
\hline Atrial Fibrillation/Flutter & $35(62.5 \%)$ & $92(58.6 \%)$ & $\mathrm{p}=0.609$ \\
\hline \multicolumn{4}{|l|}{ Chronic Kidney Disease } \\
\hline (Stage III-IV) & $27(48.2 \%)$ & $68(43.3 \%)$ & $\mathrm{p}=0.526$ \\
\hline Cardiomyopathy & & & $\mathrm{p}=0.037$ \\
\hline Ischemic & $34(60.7 \%)$ & $78(49.7 \%)$ & \\
\hline Non-Ischemic & $16(28.6 \%)$ & $72(45.9 \%)$ & \\
\hline Mixed & $6(10.7 \%)$ & $7(4.5 \%)$ & \\
\hline \multicolumn{4}{|l|}{ QRS Morphology } \\
\hline Left Bundle Branch Block & $34(60.7 \%)$ & $89(56.7 \%)$ & $\mathrm{p}=0.600$ \\
\hline Right Bundle Branch Block & $9(16.1 \%)$ & $18(11.5 \%)$ & $\mathrm{p}=0.374$ \\
\hline Ejection Fraction, $\% *$ & $27.3(14.5)$ & $25.0(13.2)$ & $\mathrm{p}=0.507$ \\
\hline
\end{tabular}

* Age and ejection fraction have skewed distributions and are presented as median (interquartile range). 
Table 3

One year outcomes among patients with RV1SI ECG pattern compared to those without showing a significant difference in the primary combined outcome.

\begin{tabular}{|lrrr|}
\hline Results at One Year & ECG Findings Present $(\mathbf{n}=56)$ & ECG Findings Absent $(\mathbf{n}=157)$ & P Value \\
\hline Primary Outcomes & & & \\
\hline Combined Outcome & $19(33.9 \%)$ & $82(52.2 \%)$ & $\mathrm{p}=0.019$ \\
\hline Death & $7(12.5 \%)$ & $20(12.7 \%)$ & $\mathrm{p}=0.963$ \\
\hline Unplanned Hospitalization & $16(28.6 \%)$ & $76(48.4 \%)$ & $\mathrm{p}=0.010$ \\
\hline LVAD/Transplant & $0(0 \%)$ & $1(0.6 \%)$ & $\mathrm{p}=0.737$ \\
\hline Secondary Outcomes & & & \\
\hline Change in LVEF & & $5.51 \% \pm 11.5$ & $\mathrm{p}=0.365$ \\
\hline
\end{tabular}

* Qualifying pre and post assessment of ejection fraction was available for 117 patients. 


\section{Table 4}

Hazard ratios for the combined primary outcome (all-cause mortality, unplanned admission, or advanced CHF therapy) at one year via Cox Regression both unadjusted and when adding potential confounders to the model showing no change in significance of optimal ECG pattern to predict the combined primary outcome.

\begin{tabular}{|lrrr|}
\hline Variables & $\beta$ & $\mathbf{9 5 \%}$ CI & p-value \\
\hline RV1SI Pattern (Unadjusted) & 0.562 & {$[0.341,0.926]$} & $\mathrm{p}=0.024$ \\
\hline RV1SI Pattern (Included in Model) & 0.465 & {$[0.275,0.786]$} & $\mathrm{p}=0.004$ \\
\hline Age at Implantation & 1.003 & {$[0.987,1.020]$} & $\mathrm{p}=0.694$ \\
\hline Gender & 1.061 & {$[0.679,1.659]$} & $\mathrm{p}=0.794$ \\
\hline Underlying LBBB & 0.674 & {$[0.454,1.002]$} & $\mathrm{p}=0.051$ \\
\hline Non-Ischemic Cardiomyopathy & 0.757 & {$[0.476,1.205]$} & $\mathrm{P}=0.241$ \\
\hline Paced QRS duration & 1.006 & {$[0.998,1.014]$} & $\mathrm{P}=0.126$ \\
\hline
\end{tabular}

